\title{
Advances in Modelling, Monitoring, and Control for Complex Industrial Systems
}

\author{
Zhiwei Gao $\mathbb{D}^{1},{ }^{1}$ Sing Kiong Nguang $\mathbb{D}^{2},{ }^{2}$ and De-Xing Kong $\mathbb{D}^{3}$ \\ ${ }^{1}$ Faculty of Engineering and Environment, University of Northumbria, Newcastle upon Tyne, NE1 8ST, UK \\ ${ }^{2}$ Department of Electrical and Computer Engineering, University of Auckland, Auckland 1010, New Zealand \\ ${ }^{3}$ Department of Mathematics, Zhejiang University, Hangzhou, China
}

Correspondence should be addressed to Zhiwei Gao; zhiwei.gao@northumbria.ac.uk

Received 23 December 2018; Accepted 24 December 2018; Published 27 February 2019

Copyright (C) 2019 Zhiwei Gao et al. This is an open access article distributed under the Creative Commons Attribution License, which permits unrestricted use, distribution, and reproduction in any medium, provided the original work is properly cited.

\section{Introduction}

Nowadays, industrial systems, such as chemical process, manufacturing process, power network, sustainable energy systems, transportation systems, wireless communication network, robotic systems, and biomedical systems, are becoming more complex, which are generally composed of a couple of interconnected systems, possess high-nonlinear and stochastic dynamics, are equipped with multiple control loops, and operate under noisy environments and varying loads. On the other hand, industrial systems are becoming more expensive, which have higher requirement for operation performance, productiveness, availability, reliability, and safety. Moreover, industry 4.0 has become the current trend of automation industries, which has great impacts on improving the reliability and operation performance of complex industrial systems. Therefore, it is paramount but challenging to develop effective techniques in modelling, monitoring, and control for complex industrial systems. As rapid developments of industrial automation, cloud and cognitive computation, internet of things, artificial intelligence, cyber-physical systems, and sensor technologies, modelling, monitoring, and control for complex industrial systems have been greatly stimulated and new techniques and applications have emerged in recent years.

This special issue aims to provide a platform for researchers and engineers to report their recent results, exchange research ideas, and overlook emerging research and application directions in modelling, monitoring, and advanced control for complex industrial systems. After a strict peer-review process, thirteen papers are selected out of forty submissions. The selected papers can be categorized into modelling and optimization for complex industrial systems, monitoring and diagnosis for complex industrial systems, and advanced control for complex industrial systems, respectively. The papers selected in this special issue will be reviewed following the categories mentioned above.

\section{Modelling and Optimization for Complex Industrial Systems}

Solid oxide fuel cells (SOFCs) are electrochemical devices converting chemical energy stored in hydrocarbon fuels into electrical energy, which are alternatives to conventional power generation schemes. It is interesting to describe dynamic behavior of a solid oxide fuel cell, which is multiinput and multioutput process in essence. In the paper entitled "Towards Reduced-Order Models of Solid Oxide Fuel Cell" authored by M. Lawrynczuk, modelling issue of a solid oxide fuel cell is investigated. By using the first-principle theory, full-order model is established firstly, and reducedmodels are then derived by using state-truncation method and residualisation model-reduction method, respectively. The effectiveness of the established models is demonstrated by using simulation studies.

Recently, fractional-order differentiation equations have received attention in modelling of complex systems. The paper entitled "Fractional-Derivative Approximation of Relaxation in Complex Systems," authored by K. M. $\mathrm{Li}$ et al., presents a system identification procedure to 
build a factional-order differential equation model to accurately describe time-dependent behavior of a class of complex systems. Caputo definition of the fractional derivative, Mattag-Leffler function, and simulated annealing optimization algorithm are used to identify the model parameters. The proposed system identification modelling algorithm is validated by using experimental data of a shelland-tube heat exchanger and a multiroom building test bed.

Four-stage helicopter main gearbox has a complex structure, and its dynamic response has a significant influence on the fatigue life of the system. As a result, it is significant to carry out modelling and dynamic analysis of the fourstage helicopter gearbox. In the paper entitled "A New Mathematical Modeling Method for Four-Stage Helicopter Main Gearbox and Dynamic Response Optimisation" authored by $\mathrm{Y}$. Chen $e t$ al., finite element method and lumped mass method are synthesized to model the overall multinode dynamic model of a four-stage helicopter main gearbox.

Logistics and supply chain networks consist in a large amount of interconnected actors with significant nonlinear and stochastics features; therefore, it is challenging to model and analyse this kind of complex industrial systems. In the paper entitled "Design of a Logistics Nonlinear System for a Complex, Multiechelon, Supply Chain Network with Uncertain Demands" authored by A. G. Campanur et al., the design problem of a four-echelon supply chain is investigated, which is represented as an inventory-location model with uncertain demand and continuous review inventory policy. A mixed integer nonlinear programming model is used to seek the optimal design of the distribution network, and a linearized integer programming model is also used to save computational cost.

In order to respond to unpredictable market changes, manufacturing industries are required to reconstruct rapidly. Discrete manufacturing has thus become the mainstream model in manufacturing industries owing to its capabilities of handling noncontinuity and configurability. In the paper entitled "Optimal Allocation Method of Discrete Manufacturing Resources for Demand Coordination between Suppliers and Customers in a Fuzzy Environment" authored by $\mathrm{W} . \mathrm{Xu}$ and $\mathrm{Y}$. Yu, a resource optimization configuration approach is presented on the basis of the customers' expectation bias and the suppliers' profit maximization under fuzzy environment. A bi-level planning model for an optimal configuration of manufacturing resources is established, and a fast nondominated sorting genetic algorithm is used to solve the model. The validity and feasibility of the model are illustrated by an example and simulations.

Automatic motion planning for industrial robots plays a crucial role in the next-generation manufacturing systems such as industrial 4.0. The paper entitled "Maximizing the Coverage of Roadmap Graph for Optimal Motion Planning" authored by J.-H. Park and T.-W. Yoon addresses an optimal algorithm to optimize a roadmap graph which can cover arbitrary morphologies of the free configuration space with maximal coverage. The proposed approach is applied to an industrial robot model, and the obtained roadmap graph is demonstrated to be effective in path-finding tests via simulation studies.

\section{Monitoring and Diagnosis for Complex Industrial Systems}

Nowadays, industrial systems are becoming more complex and expensive; thus they have higher demand and requirement on system reliability and safety. As a result, monitoring and diagnosis for complex industrial systems have been a popular research topic during the last decades. Industry 4.0 has further stimulated this research and application field.

In system of systems engineering, system integrators play key roles in forming compatible and reliable interfaces between subsystems. In the paper entitled "Interface Data Modeling to Detect and Diagnose Intersystem Faults for Designing and Integrating System of Systems" authored by K.-M. Seo and K.-P. Park, a systematic solution is proposed to identify and diagnose interface faults during the phase of the system design and integration. The presented approach is applied to an underwater shipbuilding system with 10 interfaces, and 14 fault scenarios are identified and diagnosed, leading to a reliable submarine construction.

\section{Advanced Control for Complex Industrial Systems}

Singular systems are also called DAE (differential-algebraic equation) systems, descriptor systems, generalized dynamic systems, and implicit systems, which arise in convenient and natural modelling process. Many engineering systems such as electrical engineering systems, mechanical systems, chemical systems, economic systems, and environmental systems can be modeled as singular systems. Stochastic behavior in some engineering systems such as Markovian jumping would further add the complexity of system dynamics. In the paper entitled "Estimation and Synthesis of Reachable Set for Singular Markovian Jump Systems" authored by Y. Ding and H. Lui, reachable set estimation and state-feedback control are investigated. Sufficient conditions on state/output reachable sets are developed, and state-feedback controllers are designed correspondingly.

Hysteresis, widely existing in mechanical systems, would degrade system performance and even cause system instability. Moreover, stochastic uncertainties usually occur in engineering systems, which have brought more challenges in control synthesis. In the paper entitled "Adaptive Fuzzy Control for Stochastic Pure-Feedback Nonlinear Systems with Unknown Hysteresis and External Disturbance" authored by $\mathrm{X}$. Liu et al., the tracking control problem for a class of stochastic nonlinear systems subjected to unknown hysteresis is investigated. The mean-value theorem, Nussbaum function, Lyapunov function, and back-stepping techniques are used in the analysis and design. An adaptive fuzzy controller is designed to ensure that the closed-loop system is semiglobally uniformly bounded and the tracking errors converge to a small neighborhood of the origin. The proposed control algorithms are demonstrated by simulation studies.

Vision-based control has been a mainstreaming technique in industrial robotic systems; however, visual feedback delays and uncalibrated visual parameters would limit control system performance, or even damage the system stability. The 
paper entitled "Delay-Dependent Stability in Uncalibrated Image-Based Dynamic Visual Servoing Robotic System" authored by $\mathrm{T}$. Li et al. addresses an adaptive control technique to stabilize a robotic system subjected to visual delays and uncalibrated visual parameters. The delay-dependent stability conditions are also provided to reveal the relationship between the system stability and the delayed time.

Consensus is a fundament issue of the coordinated control for multiagent systems, which has a wide application in mobile robot systems, distributed target tracking, group decision making, and so forth. Group consensus is an extension of the conventional consensus issue, which has been paid attention recently. In the paper entitled "CoupleGroup Consensus: A Class of Delayed Heterogeneous Multiagent Systems in Competitive Networks" authored by L. Ji et al., couple-group consensus is dealt with for a class of heterogeneous multiagent systems subjected to both input and communication delays. A novel distributed coordination control protocol is addressed to ensure the heterogeneous system to achieve couple-group consensus asymptotically.

Distributed engine control would be the next revolution in turbine engine control systems. Networked engine control is an intermediate distributed engine control approach, which has been paid many attentions. It is evident that transmission delays and packet dropout are inevitable during data transmission. Moreover, system performance may degrade due to component aging. As a result, how to make the system robust against transmission delays, packet dropout, and component aging is an important issue in network control systems. In the paper entitled "Networked Control System Design for Turbofan Aeroengines with Aging and Deterioration" authored by R. Li et al., a network control system design approach is proposed for a mixed two-spool turbofan aeroengine with aging and deterioration so that the system can achieve robustness against dropouts and delays as well as satisfactory dynamic performance against aging and deterioration.

With an increasing demand of top quality and variety of products, modern manufacturing industries are stimulated to automate their production processes. However, modelling and control for automated manufacturing systems are challenging due to rapid changes in global markets, unexpected events, and discrete characteristics of both cellular automata and manufacturing systems. In the paper entitled "Distributed Control of a Manufacturing System with OneDimensional Cellular Automata" authored by I. BarraganVite et al., a distributed control modelling method is proposed for an automated manufacturing process. A procedure is provided to achieve local evolution rule of the automation on the basis of the relationships among the resources and material flow in manufacturing process. The effectiveness of the proposed distributed control strategy is illustrated and demonstrated by case studies.

\section{Conclusion}

An overview of the 13 selected papers for this special issue has been presented, reflecting the most recent progress in the research field. We hope this special issue can further stimulate interest in advanced modelling, monitoring, control, and optimization for complex industrial systems from both academic societies and automation industries. More research results and practical applications on complex industrial systems are expected in the future.

\section{Conflicts of Interest}

The guest editor team does not have any conflicts of interest or private agreements with companies.

\section{Acknowledgments}

The guest editors would like to express great appreciation to all authors who supported this special issue by contributing papers. The guest editor team is deeply indebted to all reviewers for their insightful and constructive comments that helped shape this special issue. Finally, the guest editors would like to thank the research supports from the Alexander von Humboldt Foundation, the NNSFC grant (61673074), and their institutes.
Zhiwei Gao

Sing Kiong Nguang De-Xing Kong 


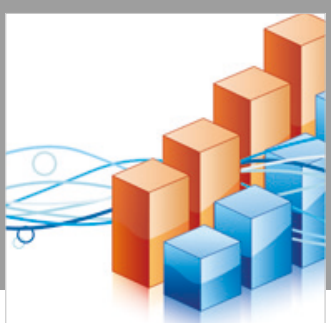

Advances in

Operations Research

\section{-n-m}
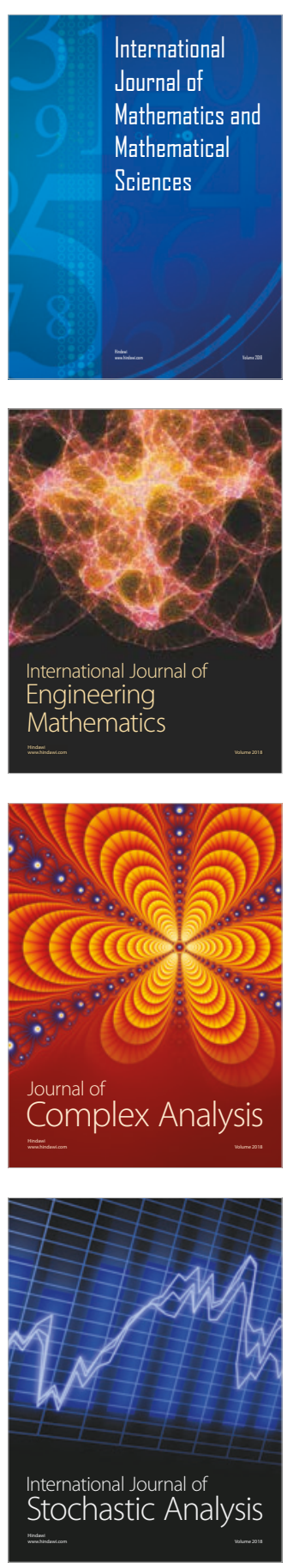
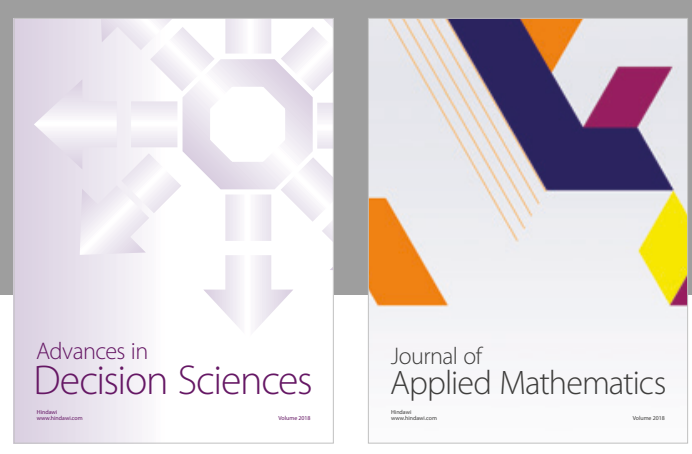

Journal of

Applied Mathematics
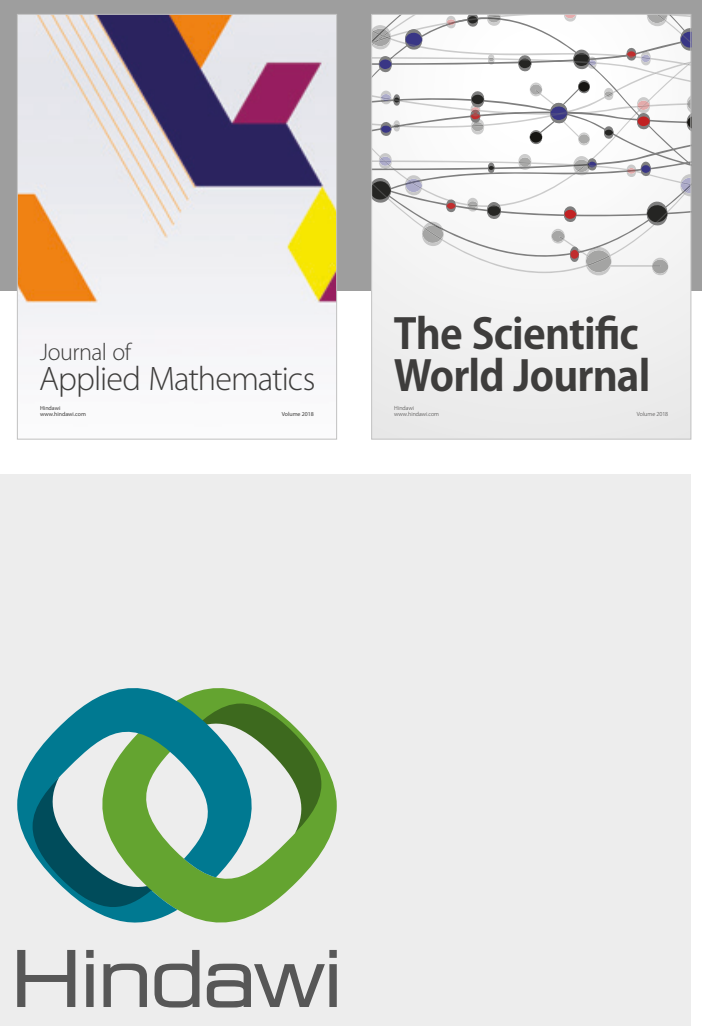

Submit your manuscripts at

www.hindawi.com

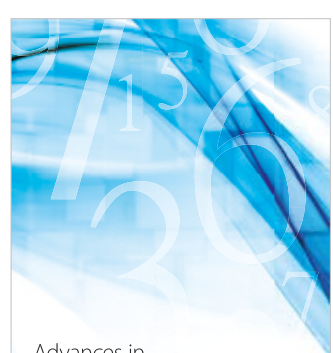

Advances in
Numerical Analysis
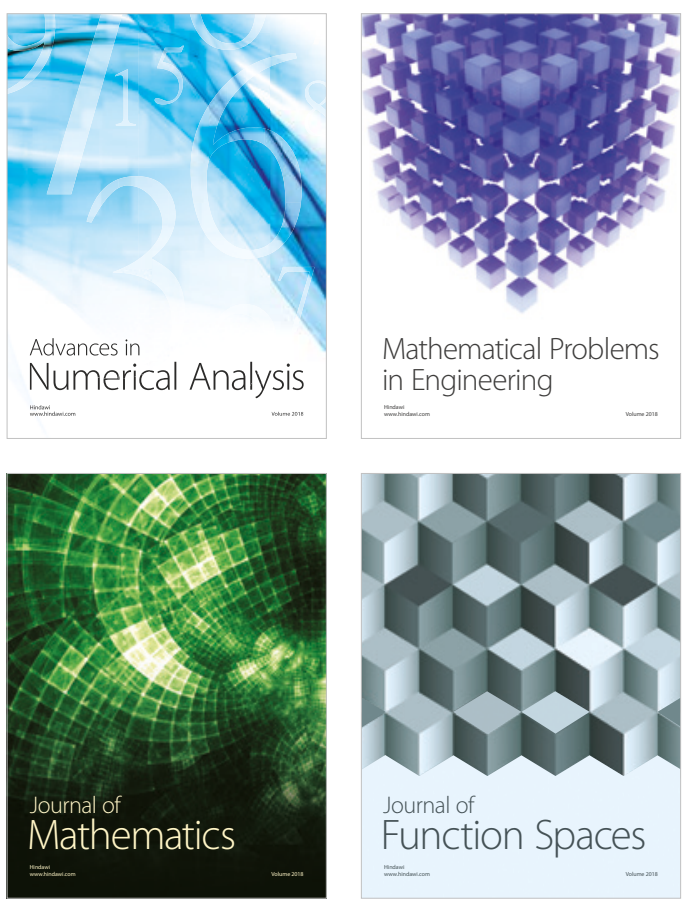

Mathematical Problems in Engineering

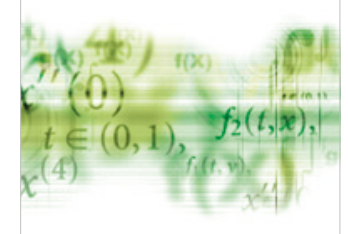

International Journal of

Differential Equations

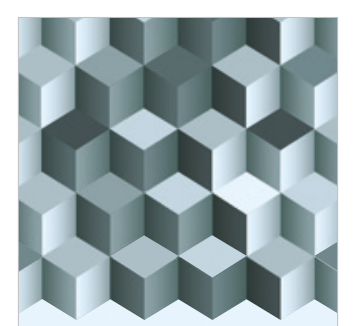

Journal of

Function Spaces
The Scientific

World Journal

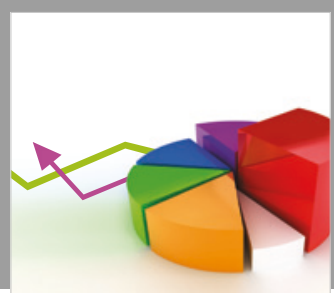

Journal of

Probability and Statistics
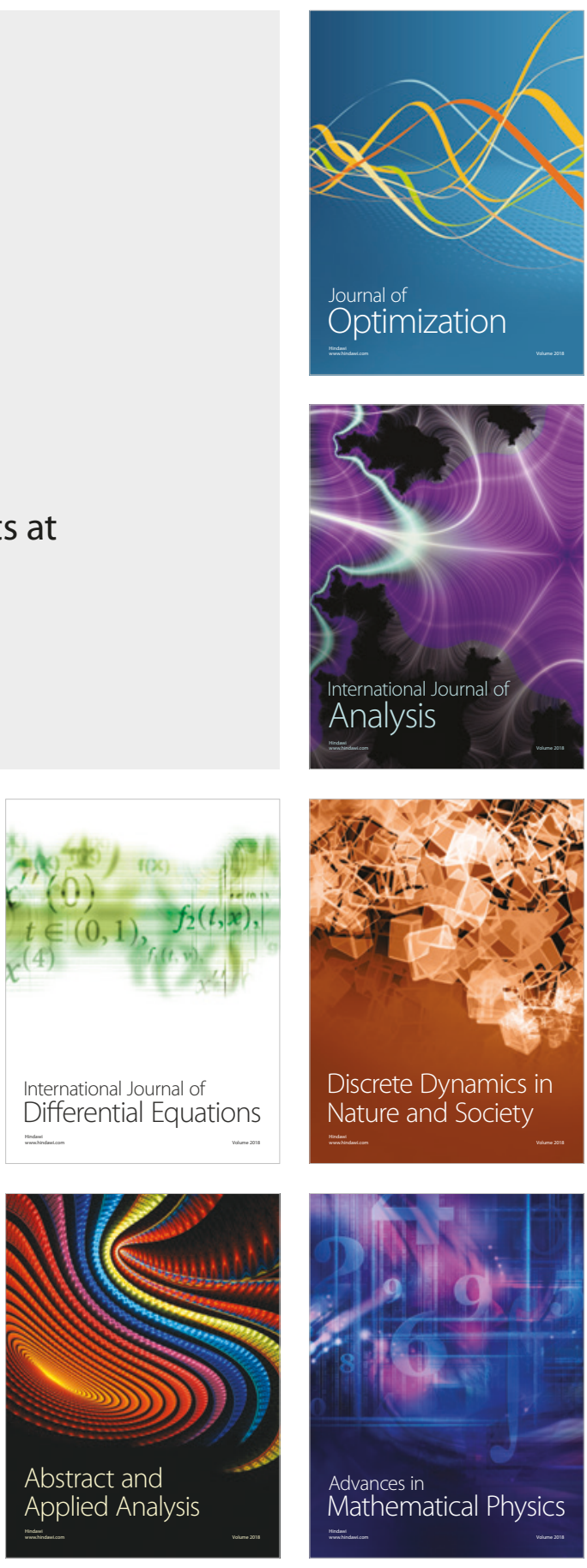